\title{
Clinical relevance of a $p$ value: Does tranexamic acid save lives after trauma or postpartum hemorrhage?
}

\author{
Geoffrey P. Dobson, PhD, FAHA, Kenji Doma, PhD, and Hayley L. Letson, PhD, \\ Townsville, Queensland, Australia
}

\section{/I Tranexamic acid could save the lives of one in three mothers who would otherwise bleed to death after childbirth." - Chief Investigators WOMAN ${ }^{I}$}

\section{Hemorrhage Control After Traumatic and Obstetric Injury}

Tranexamic acid (TXA), an antifibrinolytic agent, has been widely publicized as saving lives after traumatic hemorrhage in the CRASH-2, MATTERs, and PED-TRAX trials ${ }^{2-5}$ and more recently after postpartum hemorrhage $(\mathrm{PPH})$ in the World Maternal Antifibrinolytic (WOMAN) trial. ${ }^{6}$ Prophylactic administration of TXA is also increasingly used in major elective and emergency surgery to reduce excessive bleeding, transfusion requirements, and possible reexploration. ${ }^{7}$

Despite the claims of mortality reduction, ongoing controversies exist around the CRASH-2 trial data ${ }^{8-11}$ and more recently with the WOMAN trial data. ${ }^{6}$ Mortality reduction has been showcased on the WOMAN trial's chief investigator's institutional Web site ${ }^{1}$ and perpetuated by social media. This new strategy of communication and engagement appears to be driving a global movement among trauma surgeons and clinicians to persuade their medical advisory boards, or equivalent, to suggest that there are enough data to warrant administration of TXA in the field or clinic. It also appears to influence global health care providers and stakeholders regarding clinical, public health, or policy recommendations on TXA use.

In this current opinion, we return to the original data of both trials and address the notion that small $p$ values and large sample sizes ( $>20,000$ patients each) are sufficient to support clinical use of a drug, such as TXA. We encourage health care professionals, scientists, and governing bodies to compare the actual data with precision of the results by comparing confidence intervals (CIs). Different conclusions can be formulated from an overreliance of statistical significance ( $p$ values) without paying due attention to the magnitude of group differences. ${ }^{12} \mathrm{We}$ will first discuss the WOMAN trial followed by the CRASH-2 trial and smaller military trauma trials.

Submitted: September 28, 2017, Revised: December 14, 2017, Accepted: December 16 2017, Published online: December 28, 2017.

From the Heart, Trauma and Sepsis Research Laboratory (G.P.D., H.L.L.), College of Medicine and Dentistry, James Cook University, Townsville, Queensland, Australia; and Sport and Exercise Science (K.D.), College of Healthcare Sciences, James Cook University, Townsville, Queensland, Australia.

Address for reprints: Geoffrey Phillip Dobson, PhD, James Cook University, Townsville, Queensland, Australia; email: geoffrey.dobson@jcu.edu.au.

DOI: 10.1097/TA.0000000000001779

\section{TXA: Mechanisms and Pharmacokinetics}

Tranexamic acid is a synthetic lysine analog that can reduce active bleeding by blocking the lysine-binding sites on fibrinogen and fibrin resulting in inhibition of tissue plasminogen activator (tPA)-mediated fibrinolysis ${ }^{13}$ (Fig. 1). In some physiological states, TXA may paradoxically increase bleeding by binding to plasminogen in the presence of increased levels of urokinase plasminogen activator (uPA) and facilitate plasmin formation, which is independent of tPA ${ }^{10,14}$ (Fig. 1). The plasma half-life of TXA is approximately 2 hours, and its antifibrinolytic effects may last up to 7 to 8 hours in blood and approximately 17 hours in different tissues. ${ }^{13,15}$ Increased interest in TXA use in trauma settings and major surgery occurred 10 years ago after a newer, more potent antifibrinolytic, aprotonin, was removed from world markets. ${ }^{16,17}$ With respect to TXA's potential to reduce blood loss and mortality, it is important to note that lysine is an essential amino acid involved in multiple functions such as protein building, metabolic signaling events (via acetylation), protein-protein interactions, and posttranslational modifications in immunity and inflammation. ${ }^{18}$ It is not known how TXA affects these homeostatic functions.

\section{The WOMAN Trial Design and Main Findings}

The WOMAN trial has been heralded across social media as a breakthrough in PPH treatment driven in part by the chief investigator's institutional Web site. ${ }^{19}$ The WOMAN trial was a randomized, double-blind, placebo-controlled trial investigating the use of TXA. ${ }^{6}$ Mothers were recruited from 193 hospitals and 21 countries, mainly in Africa and Asia, but also in the United Kingdom, and the trial was funded by The Wellcome Trust and UK Department of Health through the Health Innovation Challenge Fund, as well as the Bill \& Melinda Gates Foundation.

The trial was originally planned to enroll 15,000 women with a composite primary end point of death from all causes or hysterectomy within 42 days of giving birth. ${ }^{6}$ The patient numbers were increased to more than 20,000 "in the hope that the trial would have enough power to detect a reduction in postpartum hemorrhage death," and the primary end point was revised to be a measure of "death from post-partum hemorrhage." The study reported that TXA significantly reduced the risk of death from bleeding from $1.9 \%$ (191 deaths) to $1.5 \%$ (155 deaths) $(p=0.045){ }^{6}$ Further reductions in mortality were found if TXA was given within 3 hours of giving birth (1.7-1.2\% $[p=0.008])$ or a decrease from 127 to 89 women given treatment versus placebo (in addition to standard care). ${ }^{6}$ There were 


\section{Coagulation Pathways}

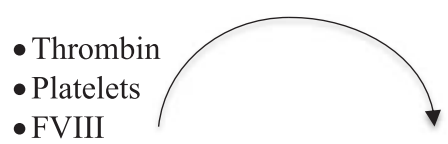

\begin{tabular}{|l|l|}
\hline Fibrinogen & Fibrin \\
\hline
\end{tabular}

\section{Fibrinolytic Pathways}
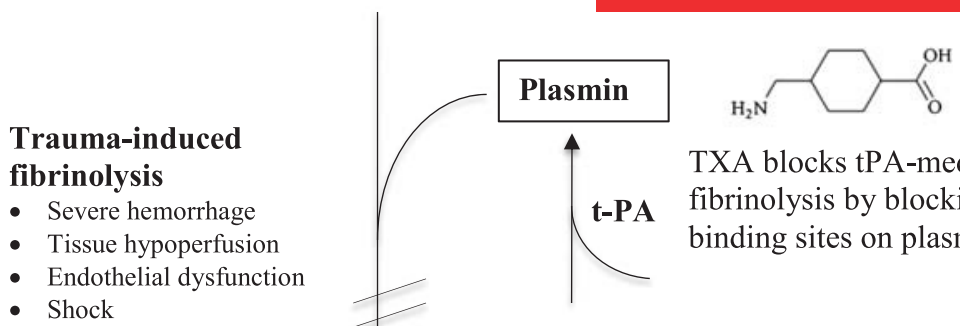

TXA blocks tPA-mediated

fibrinolysis by blocking lysine

binding sites on plasminogen

- Shock

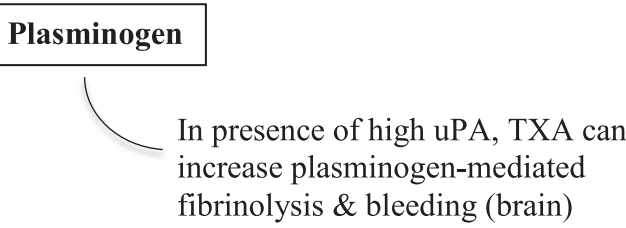

- D-Dimers

fibrinolysis \& bleeding (brain)

- Fibrin Degradation Products

Figure 1. Tranexamic acid is a synthetic derivative of the amino acid lysine that inhibits tPA-mediated fibrinolysis by blocking the five lysine-binding sites on circulating plasminogen. Circulating plasminogen is converted to the serine protease plasmin by the enzyme tPA, causing the breakdown of fibrin to D-dimers and other fibrin degradation products. Thus, TXA prevents plasmin from forming and prevents the breakdown of fibrin clots. In some cases, such as in the brain, TXA can increase bleeding by binding to plasminogen in the presence of increased levels of uPA levels, which can facilitate plasmin formation independent of tPA effect (see text).

no significant differences in reducing mortality from bleeding if TXA was given more than 3 hours after giving birth $(p=0.70)$ (Fig. 2) or from all causes $(p=0.16)$. The WOMAN Trial Collaborators concluded, "When used as a treatment for postpartum hemorrhage, TXA should be given as soon as possible

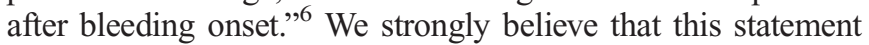
and the generalization that "tranexamic acid could save the lives of one in three mothers who would otherwise bleed to death after childbirth"1 are a gross distortion of the data and may be misleading to health professionals and the public.

\section{Relationship Between $p$ Value, CI, and Sample Size}

Fisher used the p-value as an index of evidence against the null hypothesis with this straightforward logic: when the p-value obtained from an experiment is small, then one has to assume that either an unusual event has occurred or that there is something wrong with the conditioning of the probability. Faulty conditioning would mean that the null hypothesis is not true. -Quoted from Lew ${ }^{20}$ (2012)

In the WOMAN trial, how does one interpret $p=0.008$ for mortality reduction when TXA was administered for PPH within 3 hours after childbirth when the cutoff value to reject the null hypothesis was 0.05 ? A $p$ value of 0.008 means there are eight in a thousand chances of being wrong, which sounds compelling, but there are limits to how far we can extrapolate the statistical data to TXA's lifesaving potential. In the WOMAN trial, $p=0.008$ denotes a $0.8 \%$ probability of observing a mortality difference of $0.5 \%(1.7-1.2 \%=38$ lives $)$ under the null hypothesis (that there is no effect) and would indicate the null hypothesis be rejected. However, the question of the "magnitude of difference between TXA and placebo groups" requires knowledge of the CI. ${ }^{21,22}$ The CI can be simply defined as the probability that a value will fall within the upper and lower bounds of a set probability distribution, and therefore it represents a range of values where an investigator is reasonably sure (95\% confident) that the "true value" will be found. In the WOMAN trial, the CI for $p=0.008$ was 0.52 to 0.91 , which is wide, and although it indicates a direction of effect, it lessens the strength of the data because the upper bound approaches 1.0 (Fig. 2). A wide CI also implies the sample size is too small, ${ }^{21,22}$ and given that the sample size was increased by $33 \%$ from 15,000 to 20,000 , presumably to reach significance, the data remain highly variable. To reduce variability (reduce the CI bandwidth) in half, you would have to quadruple the sample size to 80,000 patients. $^{22}$

In addition, analysis of the entire patient population who died of bleeding in the WOMAN trial also showed statistical significance between TXA and placebo groups within the 95\% CI $(p=0.045)$. However, on closer inspection, the CI of the relative risk (RR) includes the null value of $1(0.65-1.00)$, which further reduces one's confidence for a clinical effect to reduce mortality in the entire population. Therefore, the significant differences in the WOMAN trial appear to be marginal at best and do not support the generalization on the chief investigator's Web site, which went viral on social media, that "tranexamic acid could 


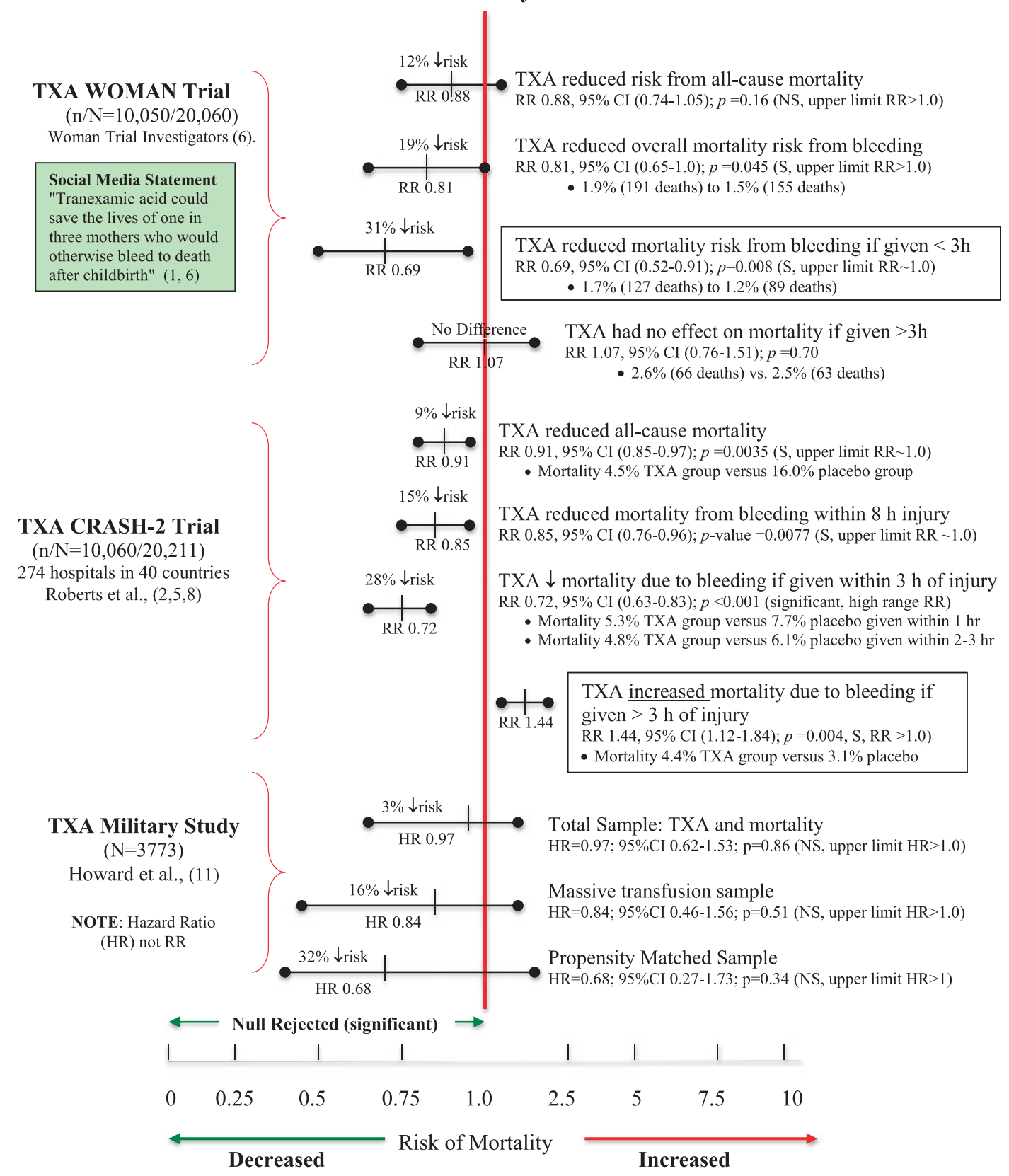

Figure 2. Schematic of the effect of TXA on mortality risk and their associated Cls in three separate studies: the WOMAN trial, CRASH-2 trial, and a military study (see text). Statistical significance in each trial was assessed using $95 \%$ Cls. In analyzing the data, the "no effect" occurs when the Cl touches 1.0 for RRs, or if the upper-bound Cl or RR is closer to 1.0; this indicates reduced clinical relevance despite a significant difference (low $p$ value). In our schematic, RR or HR values of less than 1.0 indicate increased survival, whereas greater than 1.0 indicates no change or increased risk of death. HR indicates hazard ratio; NS, not significant; $\mathrm{RR}$, risk ratio; S, significant. RR provides the cumulative risk over a time interval, whereas HR gives the instantaneous risk at a particular time point. $\mathrm{n} / \mathrm{N}$ denote the original sample size/final sample size.

save the lives of one in three mothers who would otherwise bleed to death after childbirth."

\section{Statistical Versus Clinical Significance}

Another important question that arises from the WOMAN trial is whether a $0.4 \%$ to $0.5 \%$ difference in mortality reduction from bleeding is clinically relevant. The problem, as alluded to above, is that any small difference will be statistically significant if the sample size is large enough, regardless of clinical relevance. As stated by Houle and Stump: ${ }^{23}$ "Many large clinical trials obtain a high level of statistical significance with miniscule differences between groups, which are completely clinically 
irrelevant. However, with proper marketing, billions can be made from results of dubious clinical importance."

Judgments about clinical importance should be based on the magnitude of the effect and CIs rather than a $p$-value alone, as the latter is strongly influenced by the size of the study. ${ }^{21,22}$ If the WOMAN trial kept its original sample size of 15,000 , would the results be different? As it stands, in our opinion, there appears insufficient evidence to recommend TXA's widespread use in PPH in the World Health Organization's List of Essential Medicines or treatment guidelines based solely on the WOMAN trial data alone. We offer an alternate interpretation of the WOMAN trial data:

We are $95 \%$ confident that TXA may not save the lives of one in three women who would otherwise bleed to death after childbirth. Despite showing statistically significant $p$ values in mortality reduction, the close proximity of the $R R$ to 1.0 and

upper limit of CI being close to or exceeding $1.0 \mathrm{imply}$ imprecision of the data and diminish its clinical relevance for widespread TXA use in PPH.

\section{It Is Only the Beginning: A Case for the Development of New Diagnostics and Precision-Based Medicine}

Unfortunately, the WOMAN trial did not address key clinical questions on optimal dose or whether all women should receive TXA. What was the justification for administering TXA to patients with an estimated blood loss less than $500 \mathrm{~mL}$, which falls below the normal PPH diagnostic guidelines? Of the $52 \%$ of women who lost $1 \mathrm{~L}$ or blood or less, how many died? This information was not forthcoming. Further, why was there no subgroup analysis of mortality risk based on severity of PPH? What laboratory tests are recommended to define a TXA-treatable coagulopathy with hyperfibrinolysis? What were the levels of uPA in blood of hemorrhaging women? Did any patients have fibrinolytic shutdown? Should diagnostics include plasmatic coagulation prothrombin time, activated partial thromboplastin time tests, whole-blood viscoelastic methods (thromboelastography, rotational thromboelastometry), fibrinogen levels, tPA, or tPA/plasminogen activator inhibitor 1 ratios? What clinical criteria drive TXA's use? What is the scientific basis for these clinical decisions?

Similar questions have persisted with the 2010 CRASH-2 trial, a large multicenter, randomized, and placebo-controlled trial in traumatic hemorrhage. The trial showed that TXA (1-g loading dose over 10 minutes followed by an infusion of $1 \mathrm{~g}$ over 8 hours) reduced bleeding and decreased 28-day mortality (Fig. 2) ${ }^{5,8}$ However, the trial did not show a reduction in blood transfusions, ${ }^{5,24}$ and there are ongoing controversies over TXA's safety and efficacy to reduce mortality in specific trauma populations. ${ }^{5,10}$ If administered 3 hours after hemorrhage, TXA actually increased the risk of mortality (Fig. 2). ${ }^{2,10}$ In contrast, if administered within 3 hours of injury, TXA appeared to have a survival benefit (Fig. 2).

The early survival benefit of TXA in the CRASH-2 trial was further supported by the MATTERs study, a Retrospective Observational Registry study where TXA was used with blood component-based resuscitation in combat-related trauma. ${ }^{3} \mathrm{How}-$ ever, a more recent and larger military Retrospective Observational Registry study did not support the conclusions of the
CRASH-2 trial or MATTERs study and reported no significant difference in mortality using TXA ${ }^{11}$ (Fig. 2). Despite the conflicting results on mortality reduction, there are many outstanding questions regarding the potential detrimental effects of TXA such as pulmonary embolism ${ }^{11}$ and exacerbation of secondary injury processes, including coagulopathy and inflammation. ${ }^{25}$ In a recent military retrospective cohort study, Johnston and colleagues ${ }^{26}$ reported that TXA was an independent risk factor for venous thromboembolism (odds ratio, 2.58; $95 \% \mathrm{CI}$, $1.20-5.56 ; p=0.02$ ), suggesting a reappraisal of its clinical use in combat casualties. These potential adverse effects also need to be considered for the prophylactic TXA use in pediatric $^{27}$ and adult ${ }^{25}$ elective and emergency surgeries.

On the fundamental question of survival benefit and optimal timing of TXA administration, Gayet-Ageron and colleagues $^{28}$ recently combined the CRASH-2 and WOMAN trial data and performed a meta-analysis of individual participantlevel data involving 40,138 bleeding patients. They concluded that the survival benefit of TXA was greater than $70 \%$ (odds ratio, $1.72 ; 95 \%$ CI $1.42-2.10 ; p<0.0001$ ) within the first 3 hours compared with later treatment and that the benefit decreased by $10 \%$ for every 15 minutes of treatment delay. ${ }^{28,29}$ The underlying assumptions of this meta-analysis and the validity of combining two large randomized controlled trials, each with their own controversies, and using logistic regression models to generate clinically relevant data require further evaluation. Why the meta-analysis did not include individual participant-level data from other TXA studies, such as the MATTERs, or other military studies is unfortunate. According to Gayet-Ageron and colleagues, ${ }^{28}$ any additional trials to increase patient numbers were unlikely to have "a material effect on their conclusions." However, we believe that broader inclusion criteria may help to examine the type and magnitude of potential bias in their twotrial meta-analysis. Notwithstanding these bona fide concerns, it is surprising that in October 2017 the World Health Organization revised their 2012 recommendations to include TXA within 3 hours for $\mathrm{PPH}^{30}$

In conclusion, although we agree that TXA may have an early survival benefit in patients who are severely bleeding with or without shock, ${ }^{3,31}$ the results of the WOMAN trial are only a beginning toward "saving one in three mothers who would otherwise bleed to death after childbirth," even if the drug is administered within 3 hours. We believe that the trial investigators and social media have overstated the significance of the WOMAN trial results, and combining the WOMAN and CRASH-2 data in a meta-analysis may result in inherent bias. The need for a consensus on what constitutes adequate statistical evidence for a drug to be clinically useful, and do no harm, is at the heart of our commentary. TXA should not be viewed as a one-size-fitsall approach to treat blood loss in civilian- or combat-related trauma or $\mathrm{PPH}$, but rather we propose the incorporation of a more precision-based set of guidelines for TXA administration to reduce trauma-related and maternal mortality and morbidity.

\section{KEY POINTS}

- $p$ values do not address clinical significance. 
- $p$ values represent statistical significance based on the assumption that the null hypothesis is true.

- $p$ values need to be interpreted within their confidence bandwidths (or intervals) and sample size.

- When randomized trials or observational study sample sizes are large, $p$ values can be highly significant even if the effect is small and clinically unimportant.

- Tranexamic acid should not be viewed as a one-size-fits-all approach to treat blood loss.

\section{AUTHORSHIP}

G.P.D., K.D., and H.L.L. contributed equally to the literature search, data interpretation, and writing of the manuscript.

\section{DISCLOSURE}

The authors declare no conflicts of interest.

\section{REFERENCES}

1. World Maternal Antifibrinolytic (WOMAN) Trial website. Available at: http:// womantrial.lshtm.ac.uk/. Accessed November 15, 2017.

2. CRASH-2 collaborators; Roberts I, Shakur H, Afolabi A, Brohi K, Coats T, Dewan Y, Gando S, Guyatt G, Hunt BJ, Morales C, et al. The importance of early treatment with tranexamic acid in bleeding trauma patients: an exploratory analysis of the CRASH-2 randomised controlled trial. Lancet. 2011; 377(9771):1096-1101.

3. Morrison JJ, Dubose JJ, Rasmussen TE, Midwinter MJ. Military Application of Tranexamic Acid in Trauma Emergency Resuscitation (MATTERs) Study. Arch Surg. 2012;147(2):113-119.

4. Eckert MJ, Wertin TM, Tyner SD, Nelson DW, Izenberg S, Martin MJ Tranexamic acid administration to pediatric trauma patients in a combat setting: the pediatric trauma and tranexamic acid study (PED-TRAX). $J$ Trauma Acute Care Surg. 2014;77(6):852-858.

5. Binz S, McCollester J, Thomas S, Miller J, Pohlman T, Waxman D, Shariff F, Tracy R, Walsh M. CRASH-2 study of tranexamic acid to treat bleeding in trauma patients: a controversy fueled by science and social media. $J$ Blood Transfus. 2015;2015:874920.

6. WOMAN Trial Collaborators. Effect of early tranexamic acid administration on mortality, hysterectomy, and other morbidities in women with postpartum haemorrhage (WOMAN): an international, randomised, doubleblind, placebo-controlled trial. Lancet. 2017;389(10084):2105-2116.

7. Koster A, Faraoni D, Levy JH. Antifibrinolytic therapy for cardiac surgery: an update. Anesthesiology. 2015;123(1):214-221.

8. Roberts I, Shakur H, Coats T, Hunt B, Balogun E, Barnetson L, Cook L, Kawahara T, Perel P, Prieto-Merino D, et al. The CRASH-2 trial: a randomised controlled trial and economic evaluation of the effects of tranexamic acid on death, vascular occlusive events and transfusion requirement in bleeding trauma patients. Health Technol Assess. 2013;17(10):1-79.

9. Gruen RL, Jacobs IG, Reade MC. Trauma and tranexamic acid. Research is needed to determine how patient selection and intercurrent treatment affect safety and efficacy. Med $J$ Aust. 2013;199(5):310-311.

10. Walsh M, Shreve J, Thomas S, Moore E, Moore H, Hake D, Pohlman T, Davis P, Ploplis V, Piscoya A, et al. Fibrinolysis in trauma: "myth," "reality," or "something in between". Semin Thromb Hemost. 2017;43(2): $200-212$.
11. Howard JT, Stockinger ZT, Cap AP, Bailey JA, Gross KR. Military use of TXA in combat trauma: does it matter? J Trauma Acute Care Surg. 2017; 83(4):579-588

12. McCormack J, Vandermeer B, Allan GM. How confidence intervals become confusion intervals. BMC Med Res Methodol. 2013;13:134.

13. Dunn CJ, Goa KL. Tranexamic acid: a review of its use in surgery and other indications. Drugs. 1999;57:1005-1032.

14. Medcalf RL. The traumatic side of fibrinolysis. Blood. 2015;125(16): 2457-2458.

15. Pilbrant A, Schannong M, Vessman J. Pharmacokinetics and bioavailability of tranexamic acid. Eur J Clin Pharmacol. 1981;20(1):65-72.

16. Fergusson DA, Hébert PC, Mazer CD, Fremes S, MacAdams C, Murkin JM, Teoh K, Duke PC, Arellano R, Blajchman MA, et al. A comparison of aprotinin and lysine analogues in high-risk cardiac surgery. $N$ Engl $J$ Med. 2008;358(22):2319-2331.

17. McMullan V, Alston RP. III. Aprotinin and cardiac surgery: a sorry tale of evidence misused. Br J Anaesth. 2013;110(5):675-678.

18. Draxler DF, Sashindranath M, Metcalf RL. Plasmin: a modulator of immune function. Semin Thromb Hemost. 2017;43(2):143-153.

19. Letson HL, Dobson GP. Tranexamic acid for post-partum haemorrhage in the WOMAN trial. Lancet. 2017;390(0102):1581-1582.

20. Lew MJ. Bad statistical practice in pharmacology (and other basic biomedical disciplines): you probably don't know P. Brit J Pharmacol. 2012;166: $1559-1567$.

21. Cleophas TJ. Clinical trials: renewed attention to the interpretation of the $p$ values - review. Am J Ther. 2004;11:317-322.

22. Biau DJ, Jolles BM, Porcher R. P value and the theory of hypothesis testing: an explanation for new researchers. Clin Orthop Relat Res. 2010; 468:885-892.

23. Houle TT, Stump DA. Statistical significance versus clinical significance. Semin Cardiothorac Vasc Anesth. 2008;12(1):5-6.

24. Napolitano LM, Cohen MJ, Cotton BA, Schreiber MA, Moore EE. Tranexamic acid in trauma: how should we use it? $J$ Trauma Acute Care Surg. 2013;74(6):1575-1586.

25. Sharma R, Letson HL, Smith S, Dobson GP. Tranexamic acid leads to paradoxical coagulation changes during cardiac surgery: a pilot rotational thromboelastometry study. J Surg Res. 2017;217:100-112.

26. Johnston LR, Rodriguez CJ, Elster EA, Bradley MJ. Evaluation of military use of tranexamic acid and associated thromboembolic events [published online ahead of print October 25, 2017]. JAMA Surg. 2017.

27. Maeda T, Sasabuchi Y, Matsui H, Ohnishi Y, Miyata S, Yasunaga H. Safety of tranexamic acid in pediatric cardiac surgery: a nationwide database study. J Cardiothorac Vasc Anesth. 2017;31(2):549-553.

28. Gayet-Ageron A, Prieto-Merino D, Ker K, Shakur H, Ageron FX, Roberts I. Antifibrinolytic Trials Collaboration. Effect of treatment delay on the effectiveness and safety of antifibrinolytics in acute severe haemorrhage: a meta-analysis of individual patient-level data from 40138 bleeding patients [published online ahead of print November 7, 2017]. Lancet. 2017.

29. Dries DJ. Tranexamic acid: is it about time? [published online ahead of print November 7, 2017]. Lancet. 2017.

30. Vogel JP, Oladapo OT, Dowswell T, Gülmezoglu AM. Updated WHO recommendation on intravenous tranexamic acid for the treatment of postpartum haemorrhage. Lancet Glob Health. 2018;6(1):e18-e19.

31. Cole E, Davenport R, Willett K, Brohi K. Tranexamic acid use in severely injured civilian patients and the effects on outcomes: a prospective cohort study. Ann Surg. 2015;261(2):390-394. 